\title{
RANDOM TIMES AND TIME PROJECTIONS
}

\author{
CHRIS BARNETT AND IVAN F. WILDE
}

(Communicated by Palle E. T. Jorgensen)

\begin{abstract}
Random times and their associated time projections are discussed within the context of quantum probability theory. A stochastic integral representation for time projections is obtained, and their order structure is investigated. A quantum analogue of the classical result relating the range and bounded stoppings of the stochastic integral is proved.
\end{abstract}

\section{INTRODUCTION}

In this paper we develop some of the themes introduced in [7], [8] and [5]. Within the context of certain quasi-free representations of the CAR and CCR it was shown, in [7], [8], that one can define the square of an $L^{2}$-martingale as a process in an appropriately chosen $L^{1}$-space, and this allowed one to prove that such a square has a decomposition into a martingale and an increasing process which is natural in the sense defined by Meyer [13]. In addition to this, the process obtained by stopping an $L^{2}$-martingale was characterized. In [5], it was shown that random times form a complete lattice structure and that this is, to some extent, mirrored by the corresponding family of time projections.

We shall discuss essentially three interelated topics: the structure of the time projections associated with random times, the definition of the stochastic integral with respect to an arbitrary $L^{2}$-martingale, and finally, trying the first two parts together, the characterization of the range of the stochastic integral with respect to a given $L^{2}$-martingale in terms of the time projections.

Sections 1 and 2 contain a brief review of the notions of process, random time, and the associated time projection. A time projection can be thought of as a generalization of the conditional expectation and also as the operation of stopping a process. This discussion is within the abstract setup.

In $\S \S 3,4$, and 5 , we take up in detail the quantum stochastic theory of the CAR-although all of the results have obvious analogues within the theory of the CCR. An integral representation theorem for time projections is obtained

Received by the editors July 17,1989 .

1980 Mathematics Subject Classification (1985 Revision). Primary 46L50, 60G44. 
in $\S 3$, together with a discussion of their order structure. A construction of the stochastic integral analogous to the classical construction (via the natural increasing part of the Doob-Meyer decomposition) is given in $\$ 4$. Finally, in $\S 5$, the range of the stochastic integral is related to that of the algebra generated by the time projections.

\section{NONCOMMUTATIVE PROCESSES}

The fundamental object within which we work is a noncommutative stochastic base. Specifically, we suppose that we have a von Neumann algebra $\mathfrak{A}$ on a Hilbert space $\mathscr{H}$ and an increasing family of von Neumann subalgebras $\mathfrak{A}_{t}$ indexed by $[0, \infty]$ such that $\mathfrak{A} \equiv \mathfrak{A}_{\infty}$ is generated by the collection $\mathfrak{A}_{t}: t \in[0, \infty)$. We suppose, further, that there is a unit vector $\Omega \in \mathscr{H}$ cyclic and separating for $\mathfrak{A}$ and a family $E_{t}: t \in[0, \infty]$ of normal $\omega$-invariant conditional expectations $E_{t}: \mathfrak{A} \rightarrow \mathfrak{A}_{t}$, where $\omega(\cdot)=(\cdot \Omega, \Omega)$.

Since $\Omega$ is cyclic and separating, the map $x \mapsto\|x \Omega\|, x \in \mathfrak{A}_{t}$, is a norm on $\mathfrak{A}_{t}$ and the completion is a Hilbert subspace $\mathscr{H}_{t}$ of $\mathscr{H}$, with $\mathscr{H}_{\infty}=\mathscr{H}$. We denote the orthogonal projection of $\mathscr{H}$ onto $\mathscr{H}_{t}$ by $P_{t}$. It is then easy to see that $P_{t} x \Omega=E_{t}(x) \Omega$ for $x \in \mathfrak{A}$. By analogy with the classical (commutative) theory, we sometimes write $L^{2}\left(\mathfrak{A}_{t}\right)$ for $\mathscr{H}_{t}, L^{2}(\mathfrak{A})$ for $\mathscr{H}, L^{\infty}\left(\mathfrak{A}_{t}\right)$ for $\mathfrak{A}_{t}$, and $L^{\infty}(\mathfrak{A})$ for $\mathfrak{A}$ (in these last two cases, the $\|\cdot\|_{\infty}$ norm is just the operator norm).

We define $L^{1}(\mathfrak{A})$ to be the completion of $\mathfrak{A}$ with respect to the norm

$$
x \mapsto \sup \{(y \Omega, J x \Omega): y \in \mathfrak{A},\|y\|=1\},
$$

where $J$ denotes the conjugate-linear unitary operator as given by the modular theory of $(\mathfrak{A}, \Omega)$. Then $L^{1}(\mathfrak{A}) \simeq \mathfrak{A}_{*}$. (For full details of this, see [7]). $L^{1}\left(\mathfrak{A}_{t}\right)$ is the closure of $\mathfrak{A}_{t}$ in $L^{1}(\mathfrak{A})$; thus $L^{1}\left(\mathfrak{A}_{t}\right) \subseteq L^{1}(\mathfrak{A}), t \in[0, \infty]$. One has $\mathfrak{A}_{t} \subseteq L^{2}\left(\mathfrak{A}_{t}\right) \subseteq L^{1}\left(\mathfrak{A}_{t}\right)$, for $t \in[0, \infty]$, and the conditional expectations $E_{t}$ extend to bounded maps $L^{2}(\mathfrak{A}) \rightarrow L^{2}\left(\mathfrak{A}_{t}\right)$ and $L^{1}(\mathfrak{A}) \rightarrow L^{1}\left(\mathfrak{A}_{t}\right)$, which we continue to denote by $E_{t}$. (As remarked above, the extension of $E_{t}$ to $L^{2}$ is just $P_{t}$.)

A process is a family $X_{t}$ indexed by $t \in[0, \infty)$ such that $E_{t} X_{t}=X_{t}$ for all $t$. A process $X_{t}$ is a martingale if $E_{s} X_{t}=X_{s}$ for all $0 \leq s \leq t<\infty$. Thus, we have the notion of $\mathfrak{A}$-valued, $L^{2}(\mathfrak{A})$-valued or $L^{1}(\mathfrak{A})$-valued processes and martingales. We will also think of a process as a function on $[0, \infty)$.

If $\zeta \in \mathscr{H}$, then $\left(P_{t} \zeta\right)$ is a bounded $\mathscr{H}$-valued martingale. Every bounded $\mathscr{H}$-valued martingale has this form.

Proposition 1.1. Let $\left(\zeta_{t}\right)$ be a bounded $\mathscr{H}$-valued martingale. Then there is a $\zeta \in \mathscr{H}$ such that $\zeta_{t}=P_{t} \zeta$ for all $t \in[0, \infty)$. 
Proof. Let $K=\sup \left\{\left\|\zeta_{t}\right\|: t \in[0, \infty)\right\}$. Then for fixed $\xi \in \mathscr{H}$ and $0 \leq m \leq$ $n<\infty$,

$$
\begin{aligned}
\left|\left(\zeta_{n}-\zeta_{m}, \xi\right)\right| & =\left|\left(\left(P_{n}-P_{m}\right) \zeta_{n}, \xi\right)\right| \\
& =\left|\left(\zeta_{n},\left(P_{n}-P_{m}\right) \xi\right)\right| \\
& \leq K\left\|\left(P_{n}-P_{m}\right) \xi\right\| \\
& \rightarrow 0 \text { as } m, n \rightarrow \infty
\end{aligned}
$$

since $P_{n} \uparrow \mathbf{1}$.

Hence $\left(\zeta_{n}\right)$ is weakly Cauchy, and hence there is a $\zeta \in \mathscr{H}$ such that $\zeta_{n} \rightarrow \zeta$ weakly as $n \rightarrow \infty$. Thus, for $n>t, \zeta_{t}=P_{t} \zeta_{n}$ converges weakly to $P_{t} \zeta$ as $n \rightarrow \infty$, giving $\zeta_{t}=P_{t} \zeta$.

Note that it follows that $\zeta_{t} \rightarrow \zeta$ strongly as $t \rightarrow \infty$. Thus every bounded $\mathscr{H}$ valued martingale has a 'natural' value at infinity, $\zeta_{\infty} \equiv \zeta=\lim \zeta_{t}$. Such martingales are determined completely by this value-hence we have a one-to-one correspondence between elements of $\mathscr{H}$ and bounded $\mathscr{H}$-valued martingales.

Our concern in this paper is with the noncommutative filtrations and processes for the quantum stochastic theory of the CAR and CCR [3] (for the case when the defining quasi-free state has no Fock part). As noted in [7], this also includes the Itô-Clifford theory of [2].

\section{TIME PROJECTIONS}

We recall the definitions of random time and its associated time projection. The motivation for these definitions is given in [1], [4], [5].

Definition 2.1. A random time $\tau$, adapted to the filtration $\left\{\mathfrak{A}_{t}: t \in[0, \infty]\right\}$, is an increasing projection-valued process $\left(q_{t}\right), t \in[0, \infty]$, with $q_{0}=0$ and $q_{\infty}=\mathbf{1}$.

Thus $q_{t}$ is a projection in $\mathfrak{A}_{t}$ for all $t \in \mathbf{R}^{+}$and $q_{s} \leq q_{t}$ whenever $0 \leq s \leq$ $t \leq \infty$. Note that we do not require that $\sup q_{t}=1$ as in [5].

A random time is the noncommutative counterpart to a stopping time in the classical theory.

Definition 2.2. A random time $\tau=\left(q_{t}\right)$ is called simple if it assumes only finitely many distinct values; thus $\tau=\left(q_{t}\right)$ is simple if and only if there is some finite subdivision $0=t_{0}<t_{1}<\cdots<t_{n}=\infty$ of $[0, \infty]$ such that

$$
q_{t}=\sum_{i=0}^{n-1} q_{t_{i}} \chi_{\left[t_{i}, t_{i+1}\right)}(t)
$$

for $t \in \mathbf{R}^{+}$.

Proposition 2.3. For any given random time $\tau=\left(q_{t}\right)$, there is a sequence $\tau_{n}=$ $\left(q_{t}^{(n)}\right), n=1,2, \ldots$ of simple random times such that $q_{i}^{(n)} \uparrow q_{t}$ as $n \rightarrow \infty$ for (Lebesgue) almost every $t \in \mathbf{R}^{+}$. 
Proof. The function $t \mapsto(q, \Omega, \Omega)$ is bounded and increasing on $\mathbf{R}^{+}$and so has at most countably many jumps. Since $\Omega$ is separating, it follows that $t \mapsto q_{t}$ is (Lebesgue) almost everywhere strongly continuous on $\mathbf{R}^{+}$. For each $n=1,2, \ldots$ put

$$
q_{t}^{(n)}=\sum_{k=0}^{n 2^{n}-1} q_{k / 2^{n}} \chi_{\left[k / 2^{n},(k+1) / 2^{n}\right)}(t)+\mathbf{1} \chi_{[n, \infty]}(t) .
$$

Then $\tau_{n}=\left(q_{t}^{(n)}\right)$ is a simple random time, for each $n$, and evidently $q_{t}^{(n)} \uparrow q_{t}$ as $n \rightarrow \infty$ for (Lebesgue) almost every $\mathbf{t} \in \mathbf{R}^{+}$.

Remark. By explicitly taking into account the jumps of $\left(q_{t}\right)$, one can prove that there is a sequence of simple random times which converge to $\tau$ everywhere on $\mathbf{R}^{+}$; see [1].

Definition 2.4. Let $\tau=\left(q_{t}\right)$ be a random time and let $\theta=\left\{0=t_{0}, t_{1}, \ldots, t_{n}=\right.$ $\infty\}, t_{0}<t_{1}<\cdots<t_{n}$, be a finite subdivision of $[0, \infty]$. The simple random time associated with $\tau$ and $\theta$ is that given by $\tau(\theta)=\left(q_{t}^{\theta}\right)$, where

$$
q_{t}^{\theta}=\sum_{i=0}^{n-1} q_{t_{i}} \chi_{\left(t_{i}, t_{i+1}\right)}(t)
$$

for $t \in[0, \infty)$, and $q_{\infty}^{\theta}=\mathbf{1}$.

If the family $\Theta$ of finite subdivisions of $[0, \infty]$ is partially ordered by refinement, then, as in Proposition 2.3, it follows that the net $\left\{\tau(\theta)=\left(q_{t}^{\theta}\right): \theta \in \Theta\right\}$ converges strongly (Lebesgue) almost everywhere to $\tau=\left(q_{t}\right)$.

Definition 2.5. Let $\tau^{\prime}=\left(q_{t}^{\prime}\right)$ and $\tau^{\prime \prime}=\left(q_{t}^{\prime \prime}\right)$ be random times. We say that $\tau^{\prime} \leq \tau^{\prime \prime}$ if and only if $q_{t}^{\prime} \geq q_{t}^{\prime \prime}$ for all $t \in \mathbf{R}^{+}$. We define $\tau^{\prime} \wedge \tau^{\prime \prime}$ and $\tau^{\prime} \vee \tau^{\prime \prime}$ to be the random times $\tau^{\prime} \wedge \tau^{\prime \prime}=\left(q_{t}^{\prime} \vee q_{t}^{\prime \prime}\right)$ and $\tau^{\prime} \vee \tau^{\prime \prime}=\left(q_{t}^{\prime} \wedge q_{t}^{\prime \prime}\right)$.

Thus the family of random times is partially ordered and forms a complete lattice [5].

Definition 2.6. Let $\left(\zeta_{t}\right)$ be an $L^{2}$-process and $\tau=\left(q_{t}\right)$ a random time. For $\theta=\left\{0=t_{0}, \ldots, t_{n}=\infty\right\} \in \Theta$, we define

$$
\zeta_{\tau(\theta)}=\sum_{i=0}^{n-1}\left(q_{t_{i+1}}-q_{t_{i}}\right) \zeta_{t_{i+1}} \equiv \sum_{\theta} \Delta q_{t_{i}} \zeta_{t_{i+1}}
$$

If the net $\left(\zeta_{\tau(\theta)}: \theta \in \Theta\right)$ converges in $L^{2}$ (i.e., in $\mathscr{H}$ ) then we denote the limit by $\zeta_{\tau}$ and call it the stopped process or $\zeta$ stopped by $\tau$. (Similarly, one can define stopped $L^{1}$-processes using the action of $L^{\infty}$ on $L^{1}$ (see [7]), but we shall be concerned here only with stopped $L^{2}$-processes.)

If $\left(\zeta_{t}\right)$ is an $L^{2}$-bounded martingale, then $\zeta_{t}=P_{t} \zeta$ for some $\zeta \in \mathscr{H}$, and so $\zeta_{\tau(\theta)}$ becomes

$$
\begin{aligned}
\zeta_{\tau(\theta)} & =\sum_{\theta} \Delta q_{t_{i}} P_{t_{i+1}} \zeta \\
& =M_{\tau(\theta)} \zeta
\end{aligned}
$$


where $M_{\tau(\theta)}=\sum_{i=0}^{n-1}\left(q_{t_{i+1}}-q_{t_{i}}\right) P_{t_{i+1}}$. For each $\theta \in \Theta, M_{\tau(\theta)}$ is a bounded linear operator on $\mathscr{H}$.

Theorem 2.7. For each random time $\tau$ and finite subdivision $\theta \in \Theta$, the operator $M_{\tau(\theta)}$ is an orthogonal projection on $\mathscr{H}$, and if $\sigma$ is a random time with $\tau \leq \sigma$, then $M_{\tau(\theta)} \leq M_{\sigma(\theta)}$. If $\theta_{2}$ is a refinement of $\theta_{1}$ in $\Theta$, then $M_{\tau\left(\theta_{2}\right)} \leq M_{\tau\left(\theta_{1}\right)}$.

Proof. See [5].

It follows from this theorem that for each random time $\tau$ the net $\left\{M_{\tau(\theta)}: \theta \in\right.$ $\Theta\}$ converges (strongly) to an orthogonal projection on $\mathscr{H}$, which we denote by $M_{\tau}$.

Definition 2.8. For each random time $\tau$, the orthogonal projection $M_{\tau}$ is called the time projection associated with $\tau$.

As an immediate corollary to Theorem 2.7 , we have the following:

Theorem 2.9 (Optimal Stopping). For random times $\tau, \sigma$ with $\tau \leq \sigma$, we have $M_{\tau} \leq M_{\sigma}$.

Proof. One just takes limits in Theorem 2.7 [5].

If $\zeta \in \mathscr{H}$, then Theorem 2.9 gives $M_{\tau} M_{\sigma} \zeta=M_{\tau} \zeta$. In "process" terms this becomes $\left(\zeta_{\sigma}\right)_{\tau}=\zeta_{\tau}$; i.e., optional stopping. (For more details see [4, 5].)

We shall obtain a converse to this theorem in $\S 3$ (in the proof of Corollary 3.5).

Each deterministic time $s \in[0, \infty)$ defines a random time $\hat{s}=\left(q_{t}\right)$, say, by setting $q_{t}=0$ for $0 \leq t \leq s, q_{t}=1$ for $s<t$. Then evidently $M_{s}=P_{s}$, and so we can regard time projections $M_{\tau}$ as generalizing the conditional expectation.

Let $\tau=\left(q_{t}\right)$ be a random time, $\theta \in \Theta$ and $\zeta \in \mathscr{H}$. Then we have

$$
\begin{aligned}
M_{\tau(\theta)} \zeta & \equiv \sum_{\theta} \Delta q_{t_{i}} P_{t_{i+1}} \zeta=\sum_{i=0}^{n-1}\left(q_{t_{i+1}}-q_{t_{i}}\right) P_{t_{i+1}} \zeta \\
& =q_{t_{n}} P_{t_{n}} \theta-q_{t_{0}} P_{t_{0}} \zeta-\sum_{i=0}^{n-1} q_{t_{i}}\left(P_{t_{i+1}}-P_{t_{i}}\right) \zeta \\
& =\zeta-\sum_{i=0}^{n-1} q_{t_{i}}\left(P_{t_{i+1}}-P_{t_{i}}\right) \zeta \\
& \equiv \zeta-\sum_{\theta} q_{t_{i}} \Delta P_{t_{i}} \zeta
\end{aligned}
$$

where we have used $q_{0}=0, q_{\infty}=1$ (in $\mathfrak{A}$ ), and $P_{\infty}=\mathbf{1}$ in $\mathscr{B}(\mathscr{H})$. The sum $\sum_{\theta} q_{t_{i}} \Delta P_{t_{i}} \zeta$ is the stochastic integral of the simple $\mathfrak{A}$-valued integrand $q_{t}^{\theta}$ with respect to the $L^{2}$-valued martingale $\zeta_{t}=P_{t} \zeta$. Accordingly, we shall write $M_{\tau} \zeta$ as

$$
M_{\tau} \zeta=\zeta-\int_{0}^{\infty} q_{t} d \zeta_{t}
$$


where the integral exists as a limit, in $\mathscr{H}$, of the above Riemann sums, since $M_{\tau(\theta)} \rightarrow M_{\tau}$.

But

$$
\sum_{\theta} \Delta P_{t_{i}} \zeta=\zeta-P_{0} \zeta
$$

and so we can write $M_{\tau(\theta)} \zeta$ as

$$
M_{\tau(\theta)} \zeta=P_{0} \zeta+\sum_{\theta} q_{t_{i}}^{\perp} \Delta P_{t_{i}} \zeta
$$

and thus we may write

$$
M_{\tau} \zeta=P_{0} \zeta+\int_{0}^{\infty} q_{t}^{\perp} d \zeta_{t},
$$

where, as above, the integral is the strong limit of Riemann sums.

We shall consider stochastic integral representations further in the next section (Theorem 3.2 and Corollary 3.3).

Our next result shows that the time projections associated with simple random times form a lattice.

Theorem 2.10. Let $\tau=\left(p_{t}\right)$ and $\sigma=\left(q_{t}\right)$ be random times, and let $\theta \in \Theta$. Then

$$
M_{(\sigma \vee \tau)(\theta)}=M_{\sigma(\theta)} \vee M_{\tau(\theta)}
$$

and

$$
M_{(\sigma \wedge \tau)(\theta)}=M_{\sigma(\theta)} \wedge M_{\tau(\theta)} .
$$

Proof. Suppose that $\theta=\left\{0=t_{0}, \ldots, t_{n}=\infty\right\} \in \Theta$. Then for any $\zeta \in \mathscr{H}$, we have

$$
M_{\sigma(\theta)}^{\perp} \zeta=\sum_{\theta} q_{t_{i}} \Delta P_{t_{i}} \zeta=\sum_{i=0}^{n-1} q_{t_{i}}\left(P_{t_{i+1}}-P_{t_{i}}\right) \zeta
$$

and hence

$$
\begin{aligned}
M_{\tau(\theta)}^{\perp} M_{\sigma(\theta)}^{\perp} \zeta & =\sum_{j=1}^{n-1} p_{t_{j}} \Delta P_{t_{j}}\left(\sum_{i=0}^{n-1} q_{t_{i}} \Delta P_{t_{i}} \zeta\right) \\
& =\sum_{j=0}^{n-1} \sum_{i=0}^{n-1} p_{t_{j}} \Delta P_{t_{j}} q_{t_{i}} \Delta P_{t_{i}} \zeta \\
& =\sum_{j=0}^{n-1} \sum_{i=0}^{n-1} p_{t_{j}} \Delta E_{t_{j}}\left(q_{t_{i}}\right) \Delta P_{t_{j}} \Delta P_{t_{i}} \zeta
\end{aligned}
$$

where $\Delta E_{t_{j}}=E_{t_{j+1}}-E_{t_{j}}$ and the conditional expectation is

$$
E_{t}=\sum_{j=0}^{n-1} p_{t_{j}} q_{t_{j}} \Delta P_{t_{j}} \zeta
$$

since $\Delta P_{t_{i}} \Delta P_{t_{j}}=0$ if $i \neq j$. 
It follows that

$$
\left(M_{\tau(\theta)}^{\perp} M_{\sigma(\theta)}^{\perp}\right)^{k} \zeta=\sum_{j=0}^{n-1}\left(p_{t_{j}} q_{t_{j}}\right)^{k} \Delta P_{t_{j}} \zeta
$$

for any $k=1,2, \ldots$.

Letting $k \rightarrow \infty$, we obtain

$$
\begin{aligned}
M_{\tau(\theta)}^{\perp} \wedge M_{\sigma(\theta)}^{\perp} \zeta & =\sum_{j=0}^{n-1} p_{t_{j}} \wedge q_{t_{j}} \Delta P_{t_{j}} \zeta \\
& =M_{(\tau \vee \sigma)(\theta)} \zeta .
\end{aligned}
$$

Taking orthogonal complements, we see that

$$
M_{\tau(\theta)} \vee M_{\sigma(\theta)}=M_{\tau(\theta) \vee \sigma(\theta)} .
$$

For the infimum, we begin with

$$
M_{\sigma(\theta)} \zeta=P_{0} \zeta+\sum_{i=0}^{n-1} q_{t_{i}}^{\perp} \Delta P_{t_{i}} \zeta
$$

As above, we see that

$$
\left(M_{\tau(\theta)} M_{\sigma(\theta)}\right)^{k} \zeta=P_{0} \zeta+\sum_{i=0}^{n-1}\left(p_{t_{i}}^{\perp} q_{t_{i}}^{\perp}\right)^{k} \Delta P_{t_{i}} \zeta
$$

Letting $k \rightarrow \infty$, we get

$$
\begin{aligned}
M_{\tau(\theta)} \wedge M_{\sigma(\theta)} \zeta & =P_{0} \zeta+\sum_{i=0}^{n-1} p_{t_{i}}^{\perp} \wedge q_{t_{i}}^{\perp} \Delta P_{t_{i}} \zeta \\
& =M_{(\tau \wedge \sigma)(\theta)} \zeta
\end{aligned}
$$

as required.

Corollary 2.11. For any random times $\tau, \sigma$ we have

$$
M_{\tau} \wedge M_{\sigma}=M_{\tau \wedge \sigma}
$$

Proof. We have $\tau \wedge \sigma \leq \tau$ and so $M_{\tau \wedge \sigma} \leq M_{\tau}$ (by Optional Stopping, Theorem 2.9). Similarly, $M_{\tau \wedge \sigma} \leq M_{\sigma}$. Hence $M_{\tau \wedge \sigma} \leq M_{\tau} \wedge M_{\sigma}$. On the other hand, for any $\theta \in \boldsymbol{\Theta}$,

$$
M_{\tau(\theta)} \wedge M_{\sigma(\theta)}=M_{(\tau \wedge \sigma)(\theta)}
$$

by Theorem 2.10. Hence $M_{\tau} \wedge M_{\sigma} \leq M_{\tau(\theta)} \wedge M_{\sigma(\theta)}=M_{(\tau \wedge \sigma)(\theta)}$, for all $\theta \in \Theta$, giving $M_{\tau} \wedge M_{\sigma} \leq M_{\tau \wedge \sigma}$, from which the result follows.

It is not clear whether the corresponding result for the suprema holds in general. We will see, in the next section, that it is true for the quasi-free setup, where one has integral formulae for the various time projections.

The final result of this section concerns the range of the time projection. 
Theorem 2.2. Let $\tau=\left(p_{t}\right)$ be a stopping time. Then $\zeta \in L^{2}(\mathfrak{A})$ is in the range of the time projection $M_{\tau}$ if and only if $p_{t} \zeta \in L^{2}\left(\mathfrak{A}_{t}\right)$ for all $t \in[0, \infty]$.

Proof. If $\zeta=M_{\tau} \zeta$, then

$$
\begin{aligned}
p_{t} \zeta & =p_{t} M_{\tau} \zeta=\lim _{\theta} p_{t} \sum \Delta p_{s} \zeta_{s} \\
& =\lim _{\theta} \sum p_{t} \Delta p_{s} \zeta_{s},
\end{aligned}
$$

which belongs to $L^{2}\left(\mathfrak{A}_{t}\right)$ since $\zeta_{s} \in L^{2}\left(\mathfrak{A}_{t}\right)$ for all $s \leq t$, and $p_{t} \Delta p_{s}=p_{t} \wedge$ $p_{s_{i+1}}-p_{t} \wedge p_{s_{i}} \in L^{\infty}\left(\mathfrak{A}_{t}\right)$.

Hence $p_{t} \zeta \in L^{2}\left(\mathfrak{A}_{t}\right)$ for every $t \in[0, \infty]$.

Conversely, if $p_{t} \zeta \in L^{2}\left(\mathfrak{A}_{t}\right)$ for all $t \in[0, \infty]$, then

$$
\begin{aligned}
M_{\tau(\theta)} \zeta & =\sum \Delta p_{s} \zeta_{s} \\
& =\sum \Delta p_{s} p_{s_{i+1}} \zeta_{s_{i+1}} \\
& =\sum \Delta p_{s} P_{s_{i+1}}\left(p_{s_{i+1}} \zeta\right) \\
& =\sum \Delta p_{s} p_{s_{i+1}} \zeta \\
& =\sum \Delta p_{s} \zeta \\
& =\zeta .
\end{aligned}
$$

Taking the limit gives $M_{\tau} \zeta=\zeta$.

\section{THE QUANTUM STOCHASTIC THEORY OF THE CAR}

We shall specialize now take up in detail the CAR theory as developed in [3]. Consider the gauge-invariant quasi-free representation of the CAR over $L^{2}\left(\mathbf{R}^{+}\right)$ given by the state $\omega$ with

$$
\omega\left(b^{*}(f) b(g)\right)=\int_{0}^{\infty} f(s) \overline{g(s)} \rho(s) d s,
$$

where $0<\rho<1$ almost everywhere, and $f, g \in L^{2}\left(\mathbf{R}^{+}\right)$.

Let $\mathscr{A}$ denote the CAR von Neumann algebra realized concretely on $\mathscr{H}$, the tensor product of two copies of the antisymmetric Fock space over $L^{2}\left(\mathbf{R}^{+}\right)$, so that $\omega$ is the vector state $\omega(\cdot)=(\cdot \Omega, \Omega)$, where $\Omega=\Omega_{0} \otimes \Omega_{0}, \Omega_{0}$ being the Fock vacuum vector. The conditions on $\rho$ imply that $\Omega$ is cyclic and separating for $\mathscr{A}$ on $\mathscr{H}$. For $t \geq 0$, let $\mathscr{A}_{t}$ denote the von Neumann subalgebra of $\mathscr{A}$ generated by the operators $\left\{b^{*}(f): \operatorname{supp} f \subset[0, t]\right\}$. Then there exist $\omega$-invariant normal conditional expectations $e_{t}: \mathscr{A} \rightarrow \mathscr{A}_{t}$, and these extend to conditional expectations $E_{t}: L^{1}(\mathscr{A}) \rightarrow L^{1}\left(\mathscr{A}_{t}\right)$ as described in $\S 1$. Let $b_{t}=b\left(\chi_{[0, t]}\right)$. Then $b_{t}$ and $b_{t}^{*}$ are $L^{\infty}(\mathscr{A})$-valued martingales, and one can construct quantum stochastic integrals $\int_{0}^{t} d b_{s}^{*} \xi(s)$ and $\int_{0}^{t} d b_{s} \eta(s)$ for suitable 
adapted $\mathscr{H}$-valued integrands $\xi$ and $\eta$. These stochastic integrals are themselves centered $\mathscr{H}$-valued martingales and obey isometry relations. (For the details of these results see $[3,7]$ ).

There is a converse-the martingale representation theorem: every $\mathscr{H}$-valued martingale $\zeta_{t}$, say, can be written as

$$
\zeta_{t}=\alpha \Omega+\int_{0}^{t} d b_{s}^{*} \xi(s)+\int_{0}^{t} d b_{s} \eta(s)
$$

for suitable $\alpha \in \mathbf{C}$ and integrands $\xi$ and $\eta$; and this representation is unique $[11,14]$. (The uniqueness is an easy consequence of the isometry relations.) There is a similar martingale representation theorem for bosons (see $[9,12$, 15]).

We will use the stochastic integral representation of elements of $\mathscr{H}$ (see [14, 15]) to give corresponding representations for the action of the time projections $M_{\tau}$.

Theorem 3.1. For any $\zeta \in \mathscr{H}$, there is unique $\alpha \in \mathbf{C}$ and processes $\xi \in$ $L^{2}\left(\mathbf{R}^{+},(1-\rho(s)) d s ; \mathscr{H}\right)$ and $\eta \in L^{2}\left(\mathbf{R}^{+}, \rho(s) d s ; \mathscr{H}\right)$ such that

$$
\zeta=\alpha \Omega+\int_{0}^{\infty} d b_{s}^{*} \xi(s)+\int_{0}^{\infty} d b_{s} \eta(s) .
$$

Proof. See [14].

We will write $\zeta=(\alpha, \xi, \eta)$ for notational convenience.

Theorem 3.2. Let $\tau=\left(q_{t}\right)$ be a random time and let $\zeta=(\alpha, \xi, \eta) \in \mathscr{H}$. Then

$$
M_{\tau} \zeta=\alpha \Omega+\int_{0}^{\infty} d b_{s}^{*} \beta\left(q_{s}^{\perp}\right) \xi(s)+\int_{0}^{\infty} d b_{s} \beta\left(q_{s}^{\perp}\right) \eta(s),
$$

where $\beta$ is the (spatial) parity automorphism of $\mathfrak{A}$.

Proof. Let $\left(\tau_{n}\right)=\left(\left(q_{t}^{(n)}\right)\right)$ be any sequence of simple random times such that $q_{t}^{(n)}$ converges strongly to $q_{t}$ (Lebesgue) almost everywhere, and let $\left(\xi_{m}\right)$ and $\left(\eta_{m}\right)$ be sequences of simple $\mathscr{H}$-valued processes converging to $\xi$ and $\eta$ in $L^{2}\left(\mathbf{R}^{+},(1-\rho(s)) d s ; \mathscr{H}\right)$ and $L^{2}\left(\mathbf{R}^{+}, \rho(s) d s ; \mathscr{H}\right)$, respectively.

Then $\zeta_{m}=\left(\alpha, \xi_{m}, \eta_{m}\right)$ converges to $\zeta$ in $\mathscr{H}$ as $m \rightarrow \infty$ (this is a direct consequence of the isometry relations satisfied by the stochastic integrals [3]).

Now

$$
M_{\tau_{n}} \zeta_{m}=P_{0} \zeta_{m}+\int_{0}^{\infty} q_{t}^{(n) \perp} d \zeta_{m}(t)
$$

where the integral on the right-hand side is actually a finite sum. By writing out this sum explicitly and using the definition of the stochastic integrals for simple integrands, one readily sees that

$$
M_{\tau_{n}} \zeta_{m}=\alpha \Omega+\int_{0}^{\infty} d b_{s}^{*} \beta\left(q_{s}^{(n) \perp}\right) \xi_{m}(s)+\int_{0}^{\infty} d b_{s} \beta\left(q_{s}^{(n) \perp}\right) \eta_{m}(s)
$$


As $n \rightarrow \infty$, the strong convergence of $\tau_{n}$ implies that $\beta\left(q_{s}^{(n) \perp} \xi_{m}(s) \rightarrow\right.$ $\beta\left(q_{s}^{\perp}\right) \xi_{m}(s)$ in $L^{2}\left(\mathbf{R}^{+},(1-\rho(s)) d s ; \mathscr{H}\right)$ and that $\beta\left(q_{s}^{(n) \perp}\right) \eta_{m}(s) \rightarrow \beta\left(q_{s}^{\perp}\right) \eta_{m}(s)$ in $L^{2}\left(\mathbf{R}^{+}, \rho(s) d s ; \mathscr{H}\right)$, and so

$$
M_{\tau_{n}} \zeta_{m} \rightarrow\left(\alpha, \beta\left(q_{.}^{\perp}\right) \xi_{m}, \beta\left(q_{.}^{\perp}\right) \eta_{m}\right)
$$

in $\mathscr{H}$. The limit does not depend on the particular sequence $\left(\tau_{n}\right)$. Since the net $\left\{M_{\tau(\theta)} \zeta_{m}: \theta \in \Theta\right\}$ converges to $M_{\tau} \zeta_{m}$ in $\mathscr{H}$, it follows that

$$
M_{\tau} \zeta_{m}=\left(\alpha, \beta\left(q_{.}^{\perp}\right) \xi_{m}, \beta\left(q_{.}^{\perp}\right) \eta_{m}\right) .
$$

Now, letting $m \rightarrow \infty, \beta\left(q_{.}^{\perp}\right) \xi_{m}(\cdot) \rightarrow \beta\left(q_{.}^{\perp}\right) \xi(\cdot)$ in $L^{2}\left(\mathbf{R}^{+},(1-\rho(s)) d s ; \mathscr{H}\right)$ and $\beta\left(q_{.}^{\perp}\right) \eta_{m}(\cdot) \rightarrow \beta\left(q_{.}^{\perp}\right) \eta(\cdot)$ in $L^{2}\left(\mathbf{R}^{+}, \rho(s) d s ; \mathscr{H}\right)$, and so

$$
M_{\tau} \zeta=\lim M_{\tau} \zeta_{m}=\left(\alpha, \beta\left(q_{.}^{\perp}\right) \xi, \beta\left(q_{.}^{\perp}\right) \eta\right),
$$

and the proof is complete.

Corollary 3.3. For any random time $\tau=\left(q_{t}\right)$ and any $\zeta=(\alpha, \xi, \eta)$ in $\mathscr{H}$,

$$
M_{\tau}^{\perp} \zeta=\int_{0}^{\infty} d b_{s}^{*} \beta\left(q_{s}\right) \xi(s)+\int_{0}^{\infty} d b_{s} \beta\left(q_{s}\right) \eta(s) .
$$

Proof. This follows immediately from the two previous theorems.

Corollary 3.4. The map $\tau \mapsto M_{\tau}$ is continuous in the sense that if $\left(\tau_{n}\right)$ is any sequence of random times which converge (Lebesgue) almost everywhere to the random time $\tau$, then $\left(M_{\tau_{n}}\right)$ converges strongly to $M_{\tau}$.

Proof. This follows immediately from the stochastic integral formula above and dominated convergence.

We now turn to the order structure.

Theorem 3.5. Let $\tau, \sigma$ be random times. Then

$$
M_{\sigma \vee \tau}=M_{\sigma} \vee M_{\tau} \text { and } M_{\sigma \wedge \tau}=M_{\sigma} \wedge M_{\tau} .
$$

Proof. Suppose that $\tau=\left(q_{t}\right)$ and $\sigma=\left(p_{t}\right)$. Let $\zeta=(\alpha, \xi, \eta) \in \mathscr{H}$. Then, by Theorem 3.2,

$$
\left(M_{\sigma} M_{\tau}\right)^{n} \zeta=\left(\alpha, \beta\left(\left(p_{.}^{\perp} q_{.}^{\perp}\right)^{n}\right) \xi, \beta\left(\left(p_{.}^{\perp} q_{.}^{\perp}\right)^{n}\right) \eta\right) .
$$

As $n \rightarrow \infty,\left(p_{s}^{\perp} q_{s}^{\perp}\right)^{n} \rightarrow p_{s}^{\perp} \wedge q_{s}^{\perp}$ strongly for each $s \in[0, \infty)$ and so, by dominated convergence,

$$
\beta\left(\left(p_{.}^{\perp} q_{.}^{\perp}\right)^{n}\right) \xi(\cdot) \rightarrow \beta\left(p_{.}^{\perp} \wedge q_{.}^{\perp}\right) \xi(\cdot)=\beta\left((p . \vee q .)^{\perp}\right) \xi(\cdot)
$$

in $\left.L^{2} \mathbf{R}^{+},(1-\rho(s)) d s ; \mathscr{H}\right)$, and

$$
\beta\left(\left(p_{.}^{\perp} q_{.}^{\perp}\right)^{n}\right) \eta(\cdot) \rightarrow \beta\left(p_{.}^{\perp} \wedge q_{.}^{\perp}\right) \eta(\cdot)=\beta\left((p . \vee q .)^{\perp}\right) \eta(\cdot)
$$

in $L^{2}\left(\mathbf{R}^{+}, \rho(s) d s ; \mathscr{H}\right)$. It follows that $\left(M_{\sigma} M_{\tau}\right)^{n} \zeta \rightarrow M_{\sigma \wedge \tau} \zeta$ in $\mathscr{H}$ as $n \rightarrow \infty$. But $\left(M_{\sigma} M_{\tau}\right)^{n}$ converges strongly to $M_{\sigma} \wedge M_{\tau}$, and the result follows. 
An entirely similar argument, using Corollary 3.3 , shows that

$$
\begin{aligned}
M_{\sigma}^{\perp} \wedge M_{\tau}^{\perp} \zeta & =\int_{0}^{\infty} d b_{s}^{*} \beta\left(p_{s} \wedge q_{s}\right) \xi(s)+\int_{0}^{\infty} d b_{s} \beta\left(p_{s} \wedge q_{s}\right) \eta(s) \\
& =M_{\sigma \vee \tau}^{\perp} \zeta
\end{aligned}
$$

for any $\zeta \in \mathscr{H}$. That is, $M_{\sigma}^{\perp} \wedge M_{\tau}^{\perp}=M_{\sigma \vee \tau}^{\perp}$, and so $M_{\sigma} \vee M_{\tau}=M_{\sigma \vee \tau}$.

Corollary 3.6. The lattice of time projections is complete, and the map $\tau \rightarrow M_{\tau}$ is an order sequentially continuous lattice morphism: that is, for any family $\mathscr{F}=\left\{M_{\tau}: \tau \in \Lambda\right\}$ of time projections, sup $\mathscr{F}$ and $\inf \mathscr{F}$ are time projections, and if $\Lambda$ is countable, then $\sup \mathscr{F}=M_{\text {sup } \Lambda}$ and $\inf \mathscr{F}=M_{\text {inf } \Lambda}$.

Proof. We first observe that since $\mathscr{H}$ is separable, the strong operator topology on the closed unit ball of $\mathscr{B}(\mathscr{H})$ is metrizable. Hence there is an increasing sequence $\left(M_{\tau_{n}}\right)$ in $\mathscr{F}$ converging strongly to $\sup \mathscr{F}$.

For each $n$, let $\sigma_{n}=\mathrm{V}_{m \leq n} \tau_{m}$. Then $\left(\sigma_{n}\right)$ is an increasing sequence of random times with $M_{\tau_{n}} \leq M_{\sigma_{n}}=\bigvee_{m \leq n} M_{\tau_{m}}$ for all $n$, by Theorems 2.9 and 3.5. Hence $\left(M_{\sigma_{n}}\right)$ increases to $\sup \mathscr{F}$. Let $\zeta=(\alpha, \xi, \eta) \in \mathscr{H}$. Then by Theorem 3.2, $M_{\sigma_{n}} \zeta=\left(\alpha, \beta\left(q_{.}^{(n) \perp}\right) \xi(\cdot), \beta\left(q_{.}^{(n) \perp} \eta(\cdot)\right)\right.$, where $\sigma_{n}=\left(q_{t}^{(n)}\right)$.

Write $\tau_{m}=\left(p_{t}^{(m)}\right)$. Then $q_{t}^{(n) \perp}=\bigvee_{m \leq n} p_{t}^{(m) \perp}$ increases to $\bigvee_{m} p_{t}^{(m) \perp}=p_{t}^{\perp}$, say, as $n \rightarrow \infty$, for each $t$. It follows, by dominated convergence, that $M_{\sigma_{n}} \zeta \rightarrow$ $\left(\alpha, \beta\left(p_{.}^{\perp}\right) \xi(\cdot), \beta\left(p_{.}^{\perp}\right) \eta(\cdot)\right)$ as $n \rightarrow \infty$. Thus, by Theorem 3.2, $\sup \mathscr{F}=M_{\sigma}$ where $\sigma=\left(p_{t}\right)=\bigvee_{m} \tau_{m}$.

Now $\sup \mathscr{F}=M_{\sigma}$, and so $M_{\tau} \leq M_{\sigma}$ for each $\tau \in \Lambda$; i.e., $M_{\sigma} M_{\tau}=M_{\tau}$ for each $\tau \in \Lambda$. Let $\zeta=(\alpha, \xi, \eta) \in \mathscr{H}$. Then $M_{\sigma} M_{\tau} \zeta=M_{\tau} \zeta$ gives

$$
\left(\alpha, \beta\left(p_{.}^{\perp} q_{.}^{\perp}\right) \xi(\cdot), \beta\left(p_{.}^{\perp} q_{.}^{\perp}\right) \eta(\cdot)\right)=\left(\alpha, \beta\left(q_{.}^{\perp}\right) \xi(\cdot), \beta\left(q^{\perp}\right) \eta(\cdot)\right),
$$

where $\sigma=\left(p_{t}\right), \tau=\left(q_{t}\right)$.

Taking $\xi(t)=e^{-t} \Omega \in L^{2}\left(\mathbf{R}^{+},(1-\rho(s)) d s ; \mathscr{H}\right)$, we deduce that $p_{t}^{\perp} q_{t}^{\perp}=$ $q_{t}^{\perp}$ for (Lebesgue) almost every $t \in[0, \infty)$; i.e., $q_{t}^{\perp} \leq p_{t}^{\perp}$ for almost every $t \in[0, \infty)$, for each $\tau \in \Lambda$. If $\Lambda$ is countable, it follows that $\sup _{\Lambda} q_{t}^{\perp} \leq p_{t}^{\perp}$ for almost every $t \in[0, \infty)$. Hence $\sup \Lambda \leq \sigma$.

But, on the other hand, $\tau \leq \sup \Lambda$ for each $\tau \in \Lambda$ and so $M_{\tau} \leq M_{\sup \Lambda}$ for each $\tau \in \Lambda$. Hence $\sup \mathscr{F}=\sup \left\{M_{\tau}: \tau \in \Lambda\right\} \leq M_{\text {sup } \Lambda}$. We conclude that the equality $\sup \mathscr{F}=M_{q}=M_{\text {sup } \Lambda}$ holds.

The argument for the infima is similar.

Remark. In the course of the above proof, we have proved a converse to the Optimal Stopping Theorem, Theorem 2.9; namely, that if $\sigma=\left(p_{t}\right), \tau=\left(q_{t}\right)$, and $M_{\tau} \leq M_{\sigma}$, then $p_{t} \leq q_{t}$ for (Lebesgue) almost all $t \in[0, \infty]$.

\section{Stochastic INTEGRATION}

Stochastic integration with respect to an arbitrary $L^{2}$-martingale was discussed in [7]. We wish to present, in this section, a slightly different treatment 
of this topic. We shall define stochastic integration in a manner entirely analogous to the approahc taken in classical probability via the natural increasing process of the Doob-Meyer decomposition of the (modulus) square of the $L^{2}$ martingale. We shall restrict our attention to centered $L^{2}$-martingales; that is, to those orthogonal to $\Omega$ (equivalently, those vanishing at 0 ) and denote the set of these by $\mathscr{M}_{0}^{2}$.

We recall $[7,8]$ that for any $\zeta=(0, \xi, \eta) \in \mathscr{M}_{0}^{2}$ one can define its "square" $|\zeta|^{2}$, which is an $L^{1}$-process (in fact, a submartingale) and which can be written as the sum of an $L^{1}$-martingale and an increasing $L^{1}$-process $\left(\langle\zeta, \zeta\rangle_{t}\right)$ given by

$$
\langle\zeta, \zeta\rangle_{t}(\cdot)=\int_{0}^{t}(\cdot J \xi(s), J \xi(s))(1-\rho(s)) d s+\int_{0}^{t}(\cdot J \eta(s), J \eta(s)) \rho(s) d s .
$$

Definition 4.1. Let $g$ be an elementary $\mathfrak{A}$-valued process; that is, $g$ has the form

$$
g(s)=x \chi_{[a . b)}(s)
$$

for some $0 \leq a<b$ and $x \in \mathfrak{A}_{a}$. The stochastic integral of $g$ with respect to $\zeta \in \mathscr{M}_{0}^{2}$ is

$$
\int_{0}^{\infty} g d \zeta=x\left(\zeta_{b}-\zeta_{a}\right)
$$

The stochastic integral for simple $g$ (i.e., when $g$ is a finite linear combination of elementary $\mathfrak{A}$-valued processes) is defined by linearity.

Proposition 4.2. Let $\zeta=(0, \xi, \eta) \in \mathscr{M}_{0}^{2}$. For any simple $\mathfrak{A}$-valued process $g$, we have

and

$$
\int_{0}^{\infty} g d \zeta=\int_{0}^{\infty} d b_{s}^{*} \beta(g(s)) \xi(s)+\int_{0}^{\infty} d b_{s} \beta(g(s)) \eta(s)
$$

$$
\begin{aligned}
\left\|\int_{0}^{\infty} g d \zeta\right\|_{2}^{2}= & \int_{0}^{\infty}\left(\beta\left(|g(s)|^{2}\right) \xi(s), \xi(s)\right)(1-\rho(s)) d s \\
& +\int_{0}^{\infty}\left(\beta\left(|g(s)|^{2}\right) \eta(s), \eta(s)\right) \rho(s) d s \\
= & \int_{0}^{\infty} \beta\left(|g(s)|^{2}\right) d\langle\tilde{\zeta}, \tilde{\zeta}\rangle(\mathbf{1})
\end{aligned}
$$

where $\tilde{\zeta}=(0, J \xi, J \eta)$. (Note that $L^{\infty}(\mathfrak{A})=\mathfrak{A}$ acts on $L^{1}(\mathfrak{A}) \simeq \mathfrak{A}_{*}$ by the usual left action $(z \phi)(\cdot)=\phi(\cdot z), z \in \mathfrak{A}, \phi \in \mathfrak{A}_{*}$.)

Proof. This follows readily from the construction of the stochastic integrals with respect to $b_{s}^{*}$ and $b_{s}$, the isometry relations, and the formula for the increasing process in the Doob-Meyer decomposition of $|\zeta|^{2}$. See [7] for further details.

It follows from this proposition that the stochastic integral of $\beta\left(|g|^{2}\right)$ with respect to $\langle\tilde{\zeta}, \tilde{\zeta}\rangle$, when evaluated at $\mathbf{1}$, gives rise to a quadratic form on the 
simple processes. Thus, following the classical approach (see [10], for example) we make the following definition.

Definition 4.3. For a simple $\mathfrak{A}$-valued processes $f, g$ and for a given centered $L^{2}$-martingale $\zeta \in \mathscr{M}_{0}^{2}$, we define the sesquilinear form

$$
\langle f, g\rangle=\int_{0}^{\infty} \beta\left(f^{*} g\right) d\langle\tilde{\zeta}, \tilde{\zeta}\rangle(\mathbf{1}) .
$$

The kernel of this form is a linear subspace of the linear space of simple $\mathfrak{A}$ valued processes, and so we factor by these-or, equivalently, identify processes whose difference lies in the kernel. With this identification, the set of simple processes equipped with this sesquilinear form becomes a preHilbert space and the stochastic integral with respect to $\zeta$ becomes an isometry of this space into $L^{2}(\mathfrak{A})$. We denote by $L^{1}(\zeta)$ the completion of the space of (equivalence classes of) simple processes and will continue to write $\int_{0}^{\infty} f d \zeta$ for the extension of the stochastic integral with respect to $\zeta$ to all of $L^{1}(\zeta)$. Of course, the integral with respect to $\zeta$ is an isometry of $L^{1}(\zeta)$ into $L^{2}(\mathfrak{A})$, by construction, and this characterizes the integral as a map, given its value on the elementary integrands. The process obtained by stochastic integration; namely, $\left\{\int_{0}^{t} f d \zeta: t \in \mathbf{R}^{+}\right\}$can be characterized as in [7].

We shall not address the question of identifying $L^{1}(\zeta)$ as a space of concrete processes, but refer to [7] where there are results which go some way in that direction.

In the next section, we shall investigate the range of the stochastic integral.

\section{THE TIME ALGEBRA}

In this section, we shall show that the range of the stochastic integral is given by the range of the algebra of time projections.

Definition 5.1. Let $\mathscr{T}$ denote the von Neumann algebra in $\mathscr{H}$ generated by the time projections; $\mathscr{T}=\left\{M_{\tau}: \tau \text { a random time }\right\}^{\prime \prime}$. Let $\mathscr{T}_{0}$ denote the $*$-algebra generated by finite products of time projections.

Proposition 5.2. Let $f$ be an $\mathfrak{A}$-valued process which is the (Lebesgue) almost everywhere limit in the strong operator topology of a uniformly bounded sequence of simple $\mathfrak{A}$-valued processes (this is the class $\mathscr{P}([0, \infty))$ of [7]). Then $f \in$ $L^{1}(\zeta)$ for any $\zeta \in \mathscr{M}_{0}^{2}$.

Proof. This is an application of the martingale representation theorem and dominated convergence; see [7].

Corollary 5.3. Let $f$ be as above. Then the map $\zeta \mapsto \int_{0}^{\infty} f d \zeta$ defines a bounded linear operator on $\mathscr{H}$ where $\zeta \in \mathscr{H}$ has been identified with the $L^{2}$-bounded martingale $\zeta_{t}=P_{t} \zeta, t \in[0, \infty)$. 
Proof. If $\zeta=(\alpha, \xi, \eta)$, then $\int_{0}^{\infty} f d \zeta=(0, \beta(f) \xi, \beta(f) \eta)$ by [7]. Hence, by the isometry relations,

$$
\begin{aligned}
\left\|\int_{0}^{\infty} f d \zeta\right\|_{2}^{2} & =\int_{0}^{\infty}\|\beta(f(s)) \xi(s)\|^{2}(1-\rho(s)) d s+\int_{0}^{\infty}\|\beta(f(s)) \eta(s)\|^{2} \rho(s) d s \\
& \leq\left(\sup _{s}\|f(s)\|_{\infty}\right)\|\zeta\|_{2}^{2} \text {. }
\end{aligned}
$$

Corollary 5.4. Let $T \in \mathscr{T}_{0}$. Then there is a process $f$, as above, such that

$$
T \zeta=P_{0} \zeta+\int_{0}^{\infty} f d \zeta
$$

for any $\zeta \in \mathscr{H}$ (again, $\left(\zeta_{t}\right)$ is the $L^{2}$-martingale $\left(P_{t} \zeta\right)$ ).

Proof. by linearity, it is enough to suppose that $T$ is a product of time projections, $T=M_{\tau_{1}} \cdots M_{\tau_{n}}$, where $\tau_{j}=\left(q_{t}^{(j)}\right), j=1, \ldots, n$, are random times. Then if $\zeta=(\alpha, \xi, \eta)$, we have

$$
T \zeta=\alpha \Omega+\int_{0}^{\infty} d b_{s}^{*} \beta\left(q_{s}^{(1) \perp} \cdots q_{s}^{(n) \perp}\right) \xi(s)+\int_{0}^{\infty} d b_{s} \beta\left(q_{s}^{(1) \perp} \cdots q_{s}^{(n) \perp}\right) \eta(s) .
$$

Now, $\alpha \Omega=P_{0} \zeta$ and, by Proposition 2.3 (or directly), we see that $f(\cdot)=$ $\beta\left(q^{(1) \perp} \cdots q^{(n) \perp}\right)$ is the (Lebesgue) almost everywhere strong operator limit of a uniformly bounded sequence of simple $\mathfrak{A}$-valued processes, and so the result follows.

Remark. The following theorem is the counterpart of the result from classical probability which states that the range of the stochastic integral with respect to an $L^{2}$-martingale is generated by all bounded stopping of that martingale.

Theorem 5.5. For each $\zeta \in \mathscr{H}$, the closed subspace $R(\zeta)=\left\{\int_{0}^{\infty} f d \zeta: f \in\right.$ $\left.L^{1}(\zeta)\right\}$ is equal to the closure of the subspace $\mathscr{T} \zeta=\{T \zeta: T \in \mathscr{T}\}$.

Proof. We have seen that $\mathscr{T}_{0} \zeta \subset(\zeta)$, and hence $\overline{\mathscr{T}_{0} \zeta} \subseteq R(\zeta)$. But $\overline{\mathscr{T}_{0} \zeta}=\overline{\mathscr{T} \zeta}$, since $\mathscr{T}$ is the strong closure of $\mathscr{T}_{0}$, and hence $\overline{\mathscr{T} \zeta} \subseteq R(\zeta)$.

To show that $R(\zeta) \subseteq \mathscr{T} \zeta$, let $h$ be an elementary process of the form

$$
h(s)=q \chi_{[r, t)}(s),
$$

with $0<r<t$ and where $q$ is a projection in $\mathfrak{A}_{r}$.

Define

$$
q_{s}^{\prime}=\left\{\begin{array}{ll}
0, & s<r \\
q, & r \leq s<\infty, \\
1, & s=\infty
\end{array} \quad q_{s}^{\prime \prime}= \begin{cases}0, & s<t \\
q, & t \leq s<\infty \\
1, & s=\infty .\end{cases}\right.
$$

Then $\tau^{\prime}=\left(q_{s}^{\prime}\right), \tau^{\prime \prime}=\left(q_{s}^{\prime \prime}\right)$ are random times, and

$$
\left(M_{\tau}^{\prime}-M_{\tau}^{\prime \prime}\right) \zeta=\int_{0}^{\infty} h d \zeta
$$

Hence $\int_{0}^{\infty} h d \zeta \in \mathscr{T} \zeta$. 
Now let $g(s)=x \chi_{[r, t)}(s), 0<r<t$, be an elementary process with $x \in$ $\mathfrak{A}_{r}$. Then $x$ is a linear combination of four positive operators, each of which is a norm limit of a bounded sequence $\left(y_{n}\right)$ of finite linear combinations of projections belonging to $\mathfrak{A}_{r}$ (by the spectral theorem).

By the analysis above, it follows that each $\int_{0}^{\infty} y_{n} \chi_{[r, t)} d \zeta$ belongs to $\mathscr{T} \zeta$, and so $\int_{0}^{\infty} g d \zeta \in \overline{\mathscr{T} \zeta}$. By linearity, it follows that $\int_{0}^{\infty} f d \zeta \in \overline{\mathscr{T} \zeta}$ for any simple $\mathfrak{A}$-valued process $f$ and hence, by continuity, $R(\zeta) \subseteq \overline{\mathscr{T}(\zeta)}$.

Remark. We would like to reiterate at this point that all of the results of $\S 3, \S 4$, and $\S 5$ have analogues within the quantum stochastic theory of the CCR [3]. As noted in [7], given the setup and the stochastic integral representation theorem [15], the translation from the CAR to the CCR is quite routine (and amounts to little more than replacing $\beta$, the parity automorphism, by the idenitty automorphism, and replacing $\rho(s)$ by $\gamma(s)$ and $1-\rho(s)$ by $1+\gamma(s)$ where $\gamma$ is the nonnegative function on $\mathbf{R}^{+}$which determines the two-point functions which itself determines the gauge-invariant quasi-free representation of the CCR under consideration. (In [3], [7], [15], this function was denoted by $\tau$ rather than $\gamma$. We have suggested $\gamma$ here to avoid possible notational confusion with random times.)

\section{ACKNOWLEDGMENT}

The first-named author would like to thank the Department of Mathematics at the University of Iowa for support and encouragement during the preparation of this paper.

\section{REFERENCES}

1. C. Barnett and T. Lyons, Stopping noncommutative processes, Math. Proc. Cambridge Philos. Soc. 99 (1986), 151-161.

2. C. Barnett, R. F. Streater, and I. F. Wilde, The Itô-Clifford integral, J. Funct. Anal. 48 (1982), 172-212.

3. _ Quasi-free quantum stochastic integrals for the C.A.R. and C.C.R., J. Funct. Anal. 52 (1983), 19-47.

4. C. Barnett and B. Thakrar, Time projections in a von Neumann algebra, J. Operator Theory 18 (1987), 19-31.

5. A non-commutative random stopping theorem (to appear).

6. C. Barnett and I. F. Wilde, Natural processes and Doob-Meyer decompositions over a probability gage space, J. Funct. Anal. 58 (1984), 320-334.

7. __, Quantum Doob-Meyer decompositions, J. Operator Theory 20 (1988), 133-164.

8. ings of the 2nd Heidelberg Workshop on Quantum Probability, 1988 (to appear).

9. R. Hudson and J. M. Lindsay, A non-commutative martingale representation theorem for non-Fock quantum Brownian motion, J. Funct. Anal. 61 (1985), 202-221.

10. A. U. Kussmaul, Stochastic integration and generalized martingales, Research Notes in Math., vol. 11, Pitman, 1977.

11. J. M. Lindsay, Fermion martingales, Probab. Theory Related Fields 71 (1986), 307-320. 
12. J. M. Lindsay and I. F. Wilde, On non-Fock boson stochastic integrals, J. Funct. Anal. 64 (1985), 76-82.

13. P.-A. Meyer, Probability and potentials, Blaisdell, Waltham, MA, 1966.

14. I. F. Wilde, Martingales and stochastic integrals, Quantum Probability and Applications III (L. Accardi and W. von Waldenfels, eds.), Lecture Notes in Math., vol. 1303, SpringerVerlag, 1988.

15. _ Quasi-free stochastic integral representation theorems over the CCR, Math. Proc. Cambridge Philos. Soc. 10 (1988), 383-398.

Department of Mathematics, Imperial College of Science, Technology and Medicine, LONDON SW7 2BZ, UNITED KINGDOM

Department of Mathematics, King's College, London WC2R 2LS, United Kingdom 